\title{
Comprehensive Evaluation of Transformer Condition Based on Fuzzy Grey Clustering and Variable Weight
}

\author{
Renjie Song ${ }^{1}$, Ruiying Liu ${ }^{1}$ and Lin Wang ${ }^{2}$ \\ ${ }^{1}$ Northeast Dianli University, College of Information Engineering, Jilin 132012 \\ ${ }^{2}$ State Grid Jilin Power Supply Company Information \& Telecommunication \\ Branch, Jilin 132000 \\ ISrj1963331@sina.com, 1863276967@qq.com, 22402565@sina.com
}

\begin{abstract}
Aiming at the problem that the transformer condition evaluation factors have fuzzy and gray characteristics and fixed weight $(F W)$ can not accurately evaluate the condition, we proposed a condition evaluation method for transformer based on fuzzy grey clustering and variable weight. The method is applied to evaluate transformer condition layer by layer. Firstly, the confidence degree of the association rules is introduced to determine the FW of key indicators. According to the classification of transformer state grade, the triangle whiten function is established. The grey clustering coefficient (GCC) matrix and fault layer evaluation results are obtained through grey clustering method. Then the variable weight $(V W)$ is obtained by combining variable weight synthesis mode, and then the transformer condition is evaluated by fuzzy evaluation method. The transformer test report data is carried out as case analysis and the result shows that the method we proposed can assess the transformer condition objectively and accurately.
\end{abstract}

Keywords: transformer; fuzzy grey clustering; fixed weight; variable weight; comprehensive evaluation

\section{Introduction}

Condition evaluation of electric power equipment is the key link in the process of condition maintenance. Power transformer as one of the most important power equipment, its condition evaluation result directly affects the implementation of maintenance work. Therefore, the transformer condition evaluation method has become a hot research topic [1-2].

At present, the intelligent method is used to evaluate the transformer condition in home and abroad. For example, references [3-4] proposed the grey hierarchy evaluation method. This method adopts correlation analysis to get the correlation degree between different levels, so as to assess the condition. In [5-6], neural network is used to evaluate transformer condition. First, we input transformer information, and then through training this information output the transformer condition. Fuzzy comprehensive evaluation [7-9] method is aimed at the fuzzy characteristic of transformer, using fuzzy mathematics knowledge to get the membership vector, and the condition is obtained by fuzzy composite operation. In [10-11], evidence theory is applied to the transformer condition assessment. There are some literatures through the support vector machine [12-13], matter-element theory [14] and other methods to assess the condition of the transformer.

For above researches, the weight obtained by above methods is fixed weight (FW). But these authors do not consider the problem that when the transformer is abnormal, smaller weight may result in failure to accurately reflect the transformer condition. A determinate state grade boundary is given in $[4,9,11]$, but in the actual situation, the boundary is fuzzy, this determinate boundary is not consistent with the actual situation. Transformer 
evaluation factor has the characteristics of grey and fuzziness, but literatures $[3,7,10,14]$ only consider the unilateral factor.

For above problems, we proposed the fuzzy grey clustering and variable weight (VW) method for transformer condition evaluation. Firstly, association rules (AR) is introduced to calculate the FW for each key indicator, and the grey clustering analysis (GCA) is used to get the fault layer condition, that is single condition. Then the VW coefficient is obtained by the variable weight synthesis mode, and the transformer condition is evaluated by the fuzzy evaluation method. An example analysis result shows that the evaluation method is valid and practical.

This paper gives a beginning to work in this area. Then the single condition evaluation of transformer is described in Section 2. Section 3 gives the comprehensive condition evaluation method of transformer. In Section 4, a case analysis is discussed. Conclusion remarks are finally offered in Section 5.

\section{Single Condition Evaluation of Transformer}

When faced with complex evaluation problem, the complex problem can be decomposed into several sub problems, which can simplify the complex problem and improve the accuracy of the evaluation results. The single condition evaluation of transformer is the fault layer evaluation by using GCA method. GCA method not only can avoid the clear division of state grade boundary, but also considers the grey characteristics of transformer.

\subsection{Establishment Key Indicator System}

According to reference [15] and the strong association feature between fault type and key indicators, the double model key indicator system is constructed, as shown in Figure 1. The first layer is fault layer, which is divided into 8 types of faults, i.e., $F X=\left\{F X_{1}, F X_{2}\right.$, $\left.\ldots, F X_{8}\right\}$. The second layer is key indicator layer, i.e., "Moistened insulation" $F X_{1}=\left\{F X_{1,1}\right.$, $\left.F X_{1,2}, \ldots, F X_{1,8}\right\}$.

\subsection{Condition Grade Classification and Indicator Unification}

In this paper, according to the actual operation situation, the transformer condition is divided into five grades, as shown in Table 1.

Table 1. Condition Grade Classification of Transformer

\begin{tabular}{|c|c|}
\hline Condition grade & Transformer condition description \\
\hline Good & All monitoring data are close to the initial value. \\
\hline Normal & Monitoring data are far from the attention value. \\
\hline Attention & Part monitoring data are close to the attention value. \\
\hline Abnormal & Part monitoring data are close to the warning value. \\
\hline Fault & Monitoring data exceed the prescribed warning value. \\
\hline
\end{tabular}

In order to facilitate the comparison and calculation of the key indicators, the original data will be processed, and it's controlled between $0 \sim 100$, the unified formula is:

$$
x_{i j}=\frac{x_{z}-x_{i j 0}}{x_{z}-x_{c}} \times 100
$$

Where $x_{z}$ and $x_{c}$ denote the warning value and initial value of transformer key indicator. $x_{i j 0}$ represents the measured value. When $x_{i j}<0$, lets $x_{i j}=0$, when $x_{i j}>100$, lets $x_{i j}=100$. 


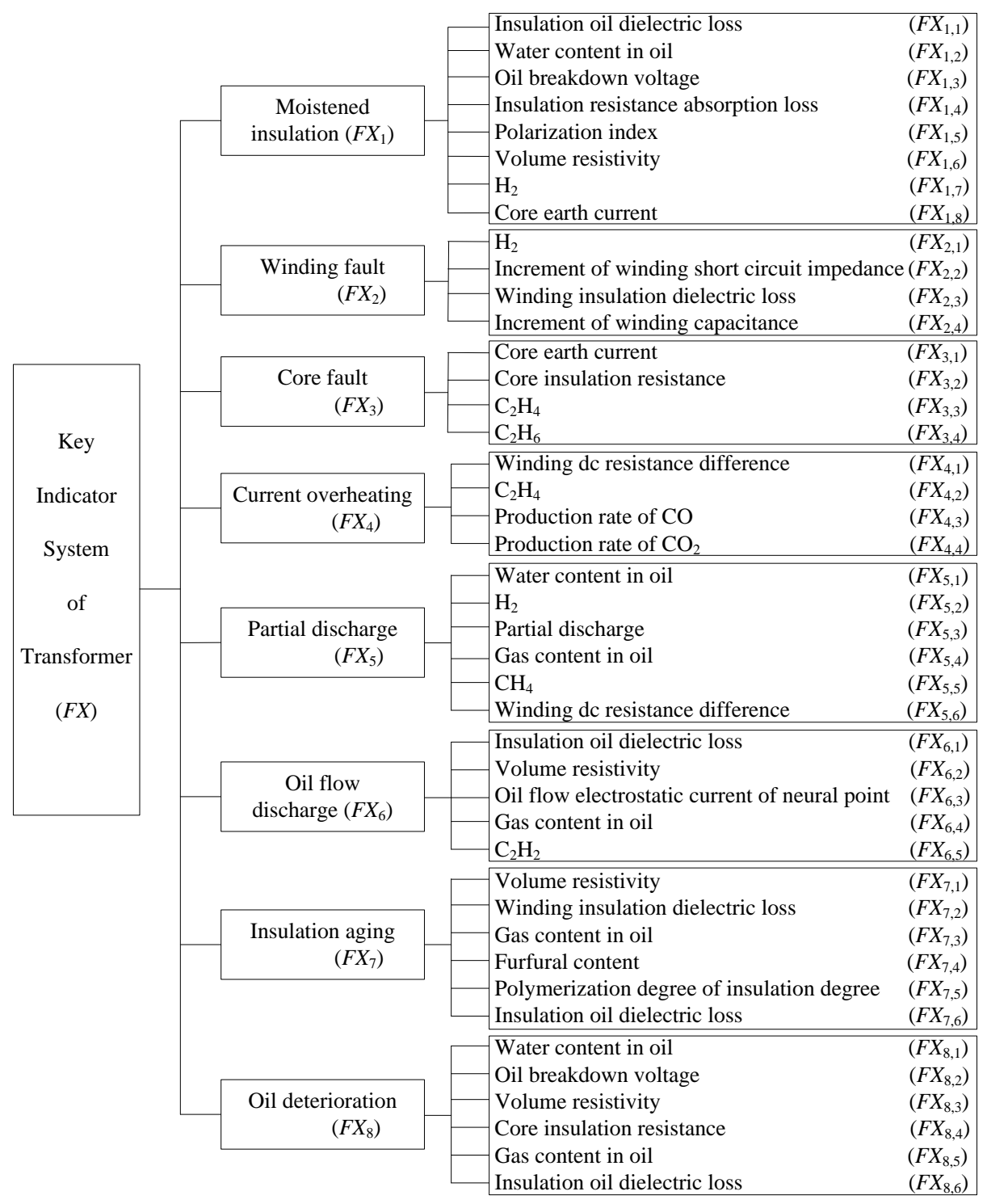

Figure 1. Key Indicator System of Transformer

\subsection{Determination of Fixed Weight}

$\mathrm{AR}$ is used to find all subsets of items or attributes, which appear frequently in the same event [16]. According to the definition of $\mathrm{AR}$, suppose $R=\left\{\alpha_{1}, \alpha_{1}, \quad, \alpha_{\mathrm{s}}\right\}$ is a finite item set which composed by $S$ item. Given the transaction database (TDB) $M=\left\{\beta_{1}, \beta_{2}, \ldots\right.$, $\left.\beta_{N}\right\}$, where $\beta_{\mathrm{i}}=\left\{\alpha_{1}, \alpha_{2}, \ldots, \alpha_{t}\right\} \subset R$, known as the t-item set. AR reflects the dependence or correlation between the different items, like $X \rightarrow Y$ [support, confidence], where $X \subseteq M, Y$ $\subseteq M$ and $X \cap Y \neq \varnothing$.

Support and confidence are two important concepts in AR. Support degree is the probability that transaction $X$ and $Y$ appear simultaneously in the TDB, that is: $S(X \rightarrow Y)=P\left(X \cup_{Y}\right)$. Confidence is the probability of both $X$ and $Y$ contained in TDB,

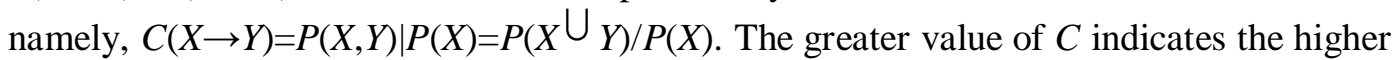
relationship between transaction $X$ and $Y$.

It is known that key indicator and fault has strong correlation, which meets the definition of confidence. Therefore, we use the confidence to calculate the FW, the steps are as follow. 
1. Determine the transaction database $M=\{$ transformer appears any fault $\}$.

2. Determine the item set $X_{i, j}=\{j$-th key indicator abnormal in $i$-th fault $\}$.

3. Determine the item set $Y_{i}=\{i$-th fault occurs $\}=M_{i}$.

4. Calculate confidence $C$ :

$$
C\left(X_{i, j} \rightarrow Y_{i}\right)=\frac{P(X \cup Y)}{P(X)}=\frac{\sigma\left(X_{i, j} \cup Y_{i}\right)}{\sigma\left(X_{i, j}\right)} \times 100 \% \text {, where } \sigma(A) \text { denotes }
$$

the number of transaction which contains $A$ in $M$.

5. Determine the FW: $\omega_{i, j}^{0}=C_{i, j} / \sum_{k=1}^{n} C_{i, k}$, which, $C_{i, j}$ represents the confidence of the $j$ th key indicator in the $i$-th fault, $n$ is the number of $\mathrm{i}$-th fault.

Each type fault of transformer is equally important to the transformer condition, so the FW of single condition is $\omega_{i}^{0}=1 / n$, in this paper $n=8$, that is $\omega_{i}^{0}=0.125$.

\subsection{Single Condition Evaluation}

The key of GCA evaluation model is to establish an effective whitening function. The evaluation model based on triangle whiten function is suitable for solving the clustering problem of less information [17]. Due to each grade boundary point of transformer is not clear, but easier to determine which grade it most likely belongs to. Therefore, this paper selects the center point triangle whiten function, and the triangle whiten function of the first grey class and final grey class is changed to the WF of lower measure and upper measure respectively. The whitening function of each grey class is fault $f_{j}^{1}$, abnormal $f_{j}^{2}$, attention $^{f_{j}^{3}}$, normal $f_{j}^{4}$, good $f_{j}^{5}$, the specific form is as follows:

$$
\begin{gathered}
f_{j}^{1}=\left\{\begin{array}{ll}
0 & x \notin[0,20] \\
1 & x \in[0,10] \\
\frac{20-x}{10} & x \in[10,20]
\end{array} f_{j}^{2}= \begin{cases}0 & x \notin[15,45] \\
\frac{x-15}{15} & x \in[15,30] \\
\frac{45-x}{15} & x \in[30,45]\end{cases} \right. \\
f_{j}^{3}= \begin{cases}0 & x \notin[35,65] \\
\frac{x-35}{15} & x \in[35,50] \\
\frac{65-x}{15} & x \in[50,65] \\
0 & f_{j}^{4}=\left\{\begin{array}{cc}
0 & x \notin[55,85] \\
\frac{x-55}{15} & x \in[55,70] \\
\frac{85-x}{15} & x \in[70,85]
\end{array}\right.\end{cases} \\
f_{j}^{5}=\left\{\begin{array}{cc}
\frac{x-80}{10} & x \in[80,100] \\
1 & x \in[80,90]
\end{array}\right.
\end{gathered}
$$

The grey clustering coefficient (GCC) of each fault is obtained by using the GCA formula $\delta_{i}^{k}=\sum_{j=1}^{m} f_{j}^{k}\left(x_{i j}\right) \omega_{i, j}^{0}$. The single condition is determined by the maximum principle method. Among them $\delta_{i}^{k}$ is GCC, ${ }^{f_{j}^{k}()}$ denotes whiten function, ${ }_{i, j}^{0}$ is FW. 


\section{Comprehensive Condition Evaluation of Transformer}

In the comprehensive condition evaluation of transformer stage, if a single condition is abnormal, and its weight coefficient is not great, it may lead to can't reflect the actual operating condition. Therefore, this paper uses the variable weight theory and fuzzy evaluation method to assess the transformer condition which can avoid the occurrence of such problems.

\subsection{Calculation of Variable Weight}

The core idea of the VW is that the VW changes with the change of state. The VW formula that introduced into the balance function is as followed:

$$
\omega_{i}\left(x_{1}, \cdots, x_{m}\right)=\omega_{i}^{0} x_{i}^{(\alpha-1)} / \sum_{k=1}^{m} \omega_{k}^{0} x_{i}^{(\alpha-1)}
$$

Which, $\omega_{i}$ and $\omega_{i}^{0}$ are the VW and FW of the $i$-th fault, $m$ denotes the number of fault, $x_{i}$ is the score of i-th fault, and $x_{i}=\omega_{i j}^{0} x_{i j}, \alpha$ denotes the balance factor. $\alpha>0.5$ indicates that the balance consideration of fault is not so important. When the serious flaw of fault is ruled out, then $\alpha<0.5$. $\alpha=1$ means equal to FW mode. In this paper $\alpha=0$.

\subsection{Comprehensive Condition Evaluation}

The comprehensive condition of transformer is obtained by using fuzzy evaluation method, the formula is $B=J \circ \omega$. Where ${ }^{\circ}$ represents the fuzzy operator, the weighted mean model is used in this paper. $J$ is the matrix of GCC, $\omega$ is VW. Finally, the maximum principle method is used to determine the transformer overall condition.

\section{Case Analysis}

\subsection{Application of Condition Evaluation Method}

Taking a transformer in a certain area as an example, the measured values $x_{i j 0}$, warning values $x_{z}$ and initial values $x_{c}$ of key indicators are shown in Table 2 .

In Figure 1 the "Winding fault $F X_{2}$ " is taken as an example to calculate the FW. We collect the data of key indicator which measured in Table 2. The total TDB contains 880 sets of data, including 116 sets of winding fault. In the 116 sets, the exceed standard times of $F X_{2,1}, F X_{2,2}, F X_{2,3}$ and $F X_{2,4}$ are 102, 112, 108 and 110. In the 880 sets of TDB, the exceed standard times of four key indicators are 396, 115, 237 and 112. That is $\sigma\left(F X_{2,1}\right)=396, \quad \sigma\left(F X_{2,2}\right)=115, \quad \sigma\left(F X_{2,3}\right)=237, \sigma\left(F X_{2,4}\right)=112, \sigma\left(F X_{2,1} \cup_{\left.F X_{2}\right)=102,} \sigma\left(F X_{2,2}\right.\right.$

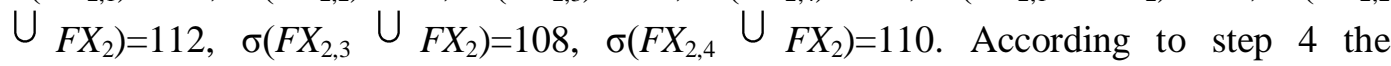
confidence of $\mathrm{H}_{2}$ is calculated,

$$
C_{2,1}=\frac{\sigma\left(F X_{2,1} \cup F X_{2}\right)}{\sigma\left(F X_{2,1}\right)} \times 100=25.76 \%
$$

$C_{2,2}=97.39 \%, C_{2,3}=45.57 \%, C_{2,4}=98.21 \%$. According to step 5 the FW is computed, $\omega_{2,1}^{0}=0.0965, \omega_{2,2}^{0}=0.3649, \omega_{2,3}^{0}=0.1707,{ }^{\omega_{2,4}^{0}}=0.3679$. In the same way, the $\mathrm{FW}$ of each key indicator is obtained, and the matrix composed of the FW is as following: 


$$
A=\left[\begin{array}{cccccccc}
0.0654 & 0.0937 & 0.1358 & 0.2632 & 0.2513 & 0.0543 & 0.0601 & 0.0762 \\
0.0965 & 0.3649 & 0.1707 & 0.3679 & - & - & - & - \\
0.3857 & 0.1574 & 0.3018 & 0.1533 & - & - & - & - \\
0.1426 & 0.1475 & 0.3513 & 0.3586 & - & - & - & - \\
0.1402 & 0.0857 & 0.2004 & 0.0859 & 0.3756 & 0.1122 & - & - \\
0.1216 & 0.1257 & 0.4413 & 0.1354 & 0.1760 & - & - & - \\
0.0917 & 0.1508 & 0.0923 & 0.2904 & 0.2980 & 0.0768 & - & - \\
0.1682 & 0.2514 & 0.1401 & 0.1805 & 0.1293 & 0.1305 & - & -
\end{array}\right]
$$

Where, $A_{i j}$ represents the $\mathrm{FW}$ of the $j$-th key indicator in $i$-th fault.

Table 2. Test Data of Key Indicator

\begin{tabular}{|c|c|c|c|}
\hline Key indicator & $\begin{array}{c}\text { Measured value } \\
x_{i j}\end{array}$ & $\begin{array}{c}\text { Warning } \\
\text { value } x_{z}\end{array}$ & $\begin{array}{c}\text { Initial } \\
\text { value } x_{c}\end{array}$ \\
\hline Oil breakdown voltage/kV & 56.7 & 27 & 58 \\
\hline Water content in oil/(mg/L) & 4.5 & 32.5 & 3.5 \\
\hline $\mathrm{H}_{2} /(\mathrm{uL} / \mathrm{L})$ & 160 & 195 & 7.0 \\
\hline Increment of winding capacitance/\% & 5.0 & 6.5 & 1 \\
\hline Furfural content/(mg/L) & 0.008 & 0.26 & 0.0 \\
\hline $\mathrm{C}_{2} \mathrm{H}_{6} /(\mathrm{uL} / \mathrm{L})$ & 19.7 & 84.5 & 2.5 \\
\hline $\mathrm{C}_{2} \mathrm{H}_{4} /(\mathrm{uL} / \mathrm{L})$ & 16.3 & 65 & 4.6 \\
\hline Gas in the oil/\% & 1.6 & 4 & 1.0 \\
\hline $\begin{array}{c}\text { impedance/\% } / \\
\text { increment winding short circuit }\end{array}$ & 3.2 & 4 & 1.0 \\
\hline Volume resistivity/(10 $\Omega$ m) & 48 & 2.3 & 60 \\
\hline Winding insulation dielectric loss/tg $\delta \%$ & 0.79 & 1.04 & 0.17 \\
\hline Winding dc resistance difference/\% & 3.7 & 5.2 & 1 \\
\hline $\mathrm{C}_{2} \mathrm{H}_{2} /(\mathrm{uL} / \mathrm{L})$ & 0 & 6.5 & 0.0 \\
\hline Insulating oil dielectric loss/tg $\delta \%$ & 1.46 & 5.2 & 0.55 \\
\hline $\mathrm{CH}_{4}$ & 27.3 & 130 & 8.5 \\
\hline Insulation resistance absorption loss/\% & 1.75 & 1 & 2 \\
\hline Polarization index & 2.16 & 1.2 & 2.4 \\
\hline Core earth current/A & 0.06 & 0.13 & 0.01 \\
\hline Core insulation resistance/M $\Omega$ & 800 & 77 & 1000 \\
\hline Production rate of CO/(\%/month) & 20 & 130 & 0 \\
\hline Production rate of CO ${ }_{2} /(\% / m o n t h)$ & 51 & 260 & 0 \\
\hline Partial discharge/pC & 67 & 650 & 30 \\
\hline $\begin{array}{c}\text { Polymerization degree of insulating } \\
\text { paper }\end{array}$ & 850 & 192 & 1000 \\
\hline $\begin{array}{c}\text { Oil flow electrostatic current of neural } \\
\text { point/uA }\end{array}$ & 0.08 & 1.3 & 0.02 \\
\hline
\end{tabular}

The data in Table 2 are put into formula (1), and then the GCC and cluster results are shown in Table 3 . The GCC matrix $J$ is shown as following: 
Table 3. GCC and Clustering Results of Single Condition

\begin{tabular}{|c|c|c|c|c|c|c|}
\hline Single condition & Fault & Abnormal & Attention & Normal & Good & Results \\
\hline Moistened insulation & 0.0088 & 0.0141 & 0.0339 & 0.3171 & 0.2323 & Normal \\
\hline Winding fault & 0.0142 & 0.7639 & 0 & 0 & 0 & Abnormal \\
\hline Core fault & 0 & 0 & 0.1723 & 0.3059 & 0.0083 & Normal \\
\hline Current overheating & 0 & 0.0878 & 0.0067 & 0.1713 & 0.1797 & Good \\
\hline Partial discharge & 0.0126 & 0.0893 & 0.0053 & 0.0286 & 0.5096 & Good \\
\hline Oil flow discharge & 0 & 0 & 0 & 0.1308 & 0.6225 & Good \\
\hline Insulation aging & 0 & 0.1381 & 0 & 0.1603 & 0.3366 & Good \\
\hline Oil deterioration & 0 & 0 & 0 & 0.2173 & 0.4252 & Good \\
\hline
\end{tabular}

$$
J=\left[\begin{array}{ccccc}
0.0088 & 0.0141 & 0.0339 & 0.3171 & 0.2323 \\
0.0142 & 0.7639 & 0 & 0 & 0 \\
0 & 0 & 0.1723 & 0.3059 & 0.0083 \\
0 & 0.0878 & 0.0067 & 0.1713 & 0.1797 \\
0.0126 & 0.0893 & 0.0053 & 0.0286 & 0.5096 \\
0 & 0 & 0 & 0.1308 & 0.6225 \\
0 & 0.1381 & 0 & 0.1603 & 0.3366 \\
0 & 0 & 0 & 0.2173 & 0.4252
\end{array}\right]
$$

According to equation (2), the VW vector is $\omega=\{0.1029,0.2995,0.1110,0.1020$, $0.1033,0.0876,0.1021,0.0916\}$. The transformer comprehensive condition is obtained by fuzzy evaluation method, and the fuzzy comprehensive evaluation results of FW and VW are shown in Table 4.

Table 4. FW and VW Fuzzy Comprehensive Evaluation Results of Transformer

\begin{tabular}{|c|c|c|c|c|c|c|}
\hline Weight mode & Fault & Abnormal & Attention & Normal & Good & Result \\
\hline Fixed weight & 0.0045 & 0.1367 & 0.0273 & 0.1664 & 0.2893 & Good \\
\hline Variable weight & 0.0065 & 0.3384 & 0.0238 & 0.1347 & 0.2236 & Abnormal \\
\hline
\end{tabular}

It can be seen from Table 4, there are significant differences between FW and VW evaluation results, and their results are "Good" and "Abnormal" respectively. But Table 3 clustering results show that the "Winding fault" is abnormal. When power-cut detection, it was discovered that the short circuit has occurred. This leads to the winding fault. Therefore, the transformer condition is abnormal. This shows that the VW fuzzy evaluation result is consistent with the actual situation. It is proved that the evaluation method proposed in this paper can accurately and objectively evaluate the transformer condition.

\subsection{Discussion}

From the clustering results of single condition, the method proposed in this paper can accurately assess the transformer single condition. This is because that we use the confidence to get the weight, and then combine with the GCA to evaluate the transformer single condition. The method avoids the problems that over-reliance on expert opinion or subjective experience and the difficulty of condition grade boundary division. The method not only gives full play to the advantages of confidence can reflect the FW of each single condition based on objective facts, but also make full use of the advantage of GCA can get each single condition, so as to evaluate the transformer single condition accurately and objectively. In the transformer comprehensive evaluation stage, it can be known that the 
VW can accurately reflect the condition of transformer through the comparison results of fixed weight and variable weight evaluation. It is because that the VW can automatically adjust the weight according to the evaluation results of single condition. From the variable weight vector, it can be seen that the single condition weight of non-normal is lager, which highlights the advantages of VW. In summary, the condition method based on fuzzy grey clustering and variable weight can accurately assess the transformer comprehensive condition. And it shows that the method has the theoretical and practical application value.

\section{Conclusions}

The contributions of this paper are summarized as following:

1. In this paper, we propose a condition evaluation method for transformer based on fuzzy grey clustering and variable weight. The fuzzy grey clustering method not only takes into account the fuzziness of the transformer system, but also considers the characteristics of its grey. The combination of FW and VW makes the evaluation method more reasonable.

2. The double key indicator system of transformer based on fault type is established. Grey clustering method is used to get the single condition of transformer, and then the VW is applied to fuzzy method to evaluate the comprehensive condition. The evaluation method of layer by layer makes the evaluation be logicality, accuracy and practicality.

3 . The experimental results show that the proposed method not only can accurately assess the status of the transformer, but also can further determine the cause of the state, which has verified the rationality and practicability of the method.

In the future work, we plan to consider more predict variables that can intervene to improve the clustering quality. And more, for a lot of data information, computer programming should be used.

\section{References}

[1] Y. Y. Zhang, R. L. Liao, L. J. Yang, F. J. Meng, J. Z. Li and H. C. Cheng, "Maintenance Decision Making of Power Transformer by Integrated Considering Reliability and Economy", Transactions of China Electrotechnical Society, vol. 29, no. 11, (2014), pp. 191-200.

[2] R. J. Liao, L. J. Yang, H. B. Zheng, K, Wang and Z. Q. Ma, "Reviews on oil-paper insulation thermal aging in power transformers", TRANSACTIONS OF CHINA ELECTROTECHNICAL SOCIETY, vol. 27, no. 5, (2012), pp. 1-12.

[3] L. Du, L. Yuan, H. Xiong, G. Tang, G, Li and C. X. Sun, "Extension Hierarchy Assessment for Operating Condition of Power Transformer", High Voltage Engineering, vol. 37, no. 4, (2011), pp. 897903.

[4] C. F. Liu, R. C. Luo, C. Y. Lei, F. F. Wang, Y. Wu and Q. Jiang, "Power Transformer Condition Assessment Based on AHP Grey Fixed-weight Clustering", Electric Power Automation Equipment, vol. 33, no. 6, (2013), pp. 104-107.

[5] Q. Yu, W. G. Li and R. C. Luo, "A risk assessment method of power transformer based on fuzzy analytic hierarchy process and neural network", Journal of Hunan University(Natural Sciences), vol. 39, no. 5, (2012), pp. 59-64.

[6] L. Ruan, Q. J. Xie, S. Y. Gao, D. X. Nie, W. H. Lu and H. L. Zhang, "Application of artificial neural network and information fusion technology in power transformer condition assessment", High Voltage Engineering, vol. 40, no. 3, (2014), pp. 822-828.

[7] Q. Yu and W. G. Li, "Application of fuzzy set pair analysis model to power transformer condition assessment”, Journal of Central South University(Science and Technology), vol. 44, no. 2, (2013), pp. 598-603.

[8] C. L. Zhang, D. Z. Wang, A. N. Wang and X. Q. Liu, "Establishment of judgment matrices for the condition assessment of the power transformer based on fuzzy AHP", Journal of Northeastern University(Natural Science), vol.34, no. 3, (2013), pp. 317-321.

[9] F. Z. Wang and L. H. Wang, "220kV transformer condition assessment strategy based on fuzzy theory", JOURNAL OF HENAN POLYTECHNIC UNIVERSITY(NATURAL SCIENCE), vol. 33, no. 3, (2014), pp. 349-353. 
[10] R. J. Liao, H. B. Zheng, S. Crzybowski, L. Yang, Y. Zhang and Y. Liao, "An integrated decisionmaking model for condition assessment of power transformers using fuzzy approach and evidential reasoning”, Strategic Management Journal, vol. 26, no. 2, (2011), pp. 1111-1118.

[11] R. J. Liao, F. J. Meng, N. R. Zhou, H. H. Xia, Y. Y. Zhang and H. C. Cheng, "Assessment Strategy for Inner Insulation Condition of Power Transformer Based on Set-pair Analysis and Evidential Reasoning Decision-making", High Voltage Engineering, vol. 40, no. 2, (2014), pp. 474-481.

[12] A. D. Ashkezari, H, Ma, T. K. Saha and C. Ekanayake, "Application of fuzzy support vector machine for determining the health index of the insulation system of in-Service power transformers", IEEE Transactions on Dielectrics and Electrical Insulation, vol. 20, no. 3, (2013), pp. 965-973.

[13] W. G. Chen, L. Teng, J. Liu, S. Y. Peng and C. X. Sun, "Transformer winding hot-spot temperature prediction model of support vector machine optimized by genetic algorithm", TRANSACTIONS OF CHINA ELECTROTECHNICAL SOCIETY, vol. 29, no. 1, (2014), pp. 44-51.

[14] L. X. Yang, F. W. Yu and Y. Bao, "Classification evaluation of transformer insulation condition based on matter-element theory", Electric Power Automation Equipment, vol. 30, no. 6, (2010), pp. 55-59.

[15] State Grid Corp of China. Q_GDW-169-2008. Guide for condition evaluation of oil-immersed power transformers (reactors), (2008).

[16] Y. J. Yan, G. H. Sheng, Y. F. Chen, Z. H. Guo, X. M. Du and Q. Wang, "Establishment of key parameter system for condition evaluation of transmission line based on association rules and principal component analysis", High Voltage Engineering, vol. 41, no. 7, (2015), pp. 2308-2314.

[17] S. F. Liu, B. Xu, J. Forrest, Y. Chen and Y. Yang, "On uniform effect measure functions and a weighted multi-attribute grey target decision model”, Journal of Grey System, vol. 25, no. 1, (2013), pp. 1-11.

\section{Authors}

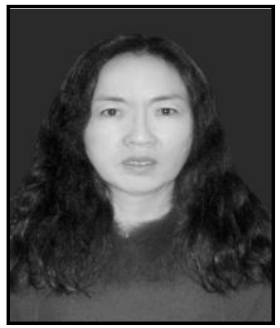

Renjie Song, she is a professor at the College of Information Engineering, Northeast Dianli University. Her research interests include application of artificial intelligence in electric power system and condition evaluation of power equipment.

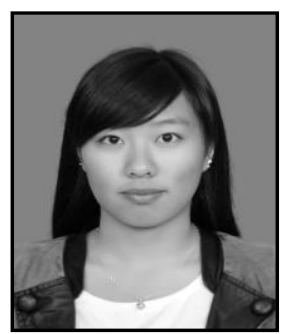

Ruiying Liu, she is a master at the College of Information Engineering, Northeast Dianli University. Her major is computer science and technology. Her research interests include condition evaluation of power equipment and application of artificial intelligence in electric power system. 
International Journal of Multimedia and Ubiquitous Engineering Vol.12, No.4 (2017) 